\title{
Associations of renin-angiotensin system genetic polymorphisms and clinical course after aneurysmal subarachnoid hemorrhage
}

\author{
Christoph J. Griessenauer, MD, ${ }^{1-3}$ R. Shane Tubbs, PhD, PA-C, ${ }^{2}$ Paul M. Foreman, MD, ${ }^{3}$ \\ Michelle H. Chua, BS, ${ }^{4}$ Nilesh A. Vyas, MD, ${ }^{5}$ Robert H. Lipsky, PhD, ${ }^{5,6}$ Mingkuan Lin, PhD, ${ }^{6}$ \\ Ramaswamy lyer, PhD, ${ }^{7}$ Rishikesh Haridas, MS, ${ }^{7}$ Beverly C. Walters, MD, MSc, FRCSC, ${ }^{3,5}$ \\ Salman Chaudry, BS, ${ }^{6}$ Aisana Malieva, BS, ${ }^{6}$ Samantha Wilkins, MA, ${ }^{6}$ Mark R. Harrigan, MD, ${ }^{3}$ \\ Winfield S. Fisher III, MD, ${ }^{3}$ and Mohammadali M. Shoja, MD²
}

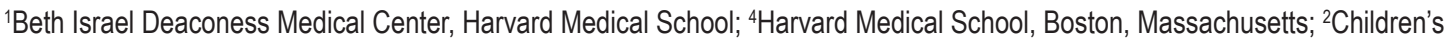
of Alabama; ${ }^{3}$ Department of Neurosurgery, University of Alabama at Birmingham, Alabama; ${ }^{5}$ Department of Neurosciences and ${ }^{7}$ Inova Translational Medicine Institute, Inova Health System, Falls Church; and ${ }^{6}$ Department of Molecular Neuroscience, George Mason University, Fairfax, Virginia

OBJECTIVE Renin-angiotensin system (RAS) genetic polymorphisms are thought to play a role in cerebral aneurysm formation and rupture. The Cerebral Aneurysm Renin Angiotensin System (CARAS) study prospectively evaluated associations of common RAS polymorphisms and clinical course after aneurysmal subarachnoid hemorrhage (aSAH).

METHODS The CARAS study prospectively enrolled aSAH patients at 2 academic centers in the United States. A blood sample was obtained from all patients for genetic evaluation and measurement of plasma angiotensin converting enzyme (ACE) concentration. Common RAS polymorphisms were detected using 5 'exonuclease genotyping assays and pyrosequencing. Analysis of associations of RAS polymorphisms and clinical course after aSAH were performed.

RESULTS A total of 166 patients were screened, and 149 aSAH patients were included for analysis. A recessive effect of allele I (insertion) of the ACE I/D (insertion/deletion) polymorphism was identified for Hunt and Hess grade in all patients (OR 2.76, 95\% Cl 1.17-6.50; $p=0.0206$ ) with subsequent poor functional outcome. There was a similar effect on delayed cerebral ischemia $(\mathrm{DCl})$ in patients 55 years or younger (OR 3.63, 95\% Cl 1.04-12.7; $p=0.0439)$. In patients older than 55 years, there was a recessive effect of allele $A$ of the angiotensin II receptor Type 2 (AT2) A/C single nucleotide polymorphism (SNP) on DCl (OR 4.70, 95\% Cl 1.43-15.4; $p=0.0111)$.

CONCLUSIONS Both the ACE I/D polymorphism and the AT2 A/C single nucleotide polymorphism were associated with an age-dependent risk of delayed cerebral ischemia, whereas only the ACE I/D polymorphism was associated with poor clinical grade at presentation. Further studies are required to elucidate the relevant pathophysiology and its potential implication in the treatment of patients with aSAH.

https://thejns.org/doi/abs/10.3171/2016.4.JNS16409

KEY WORDS renin; angiotensin; aneurysm; rupture; subarachnoid hemorrhage; vascular disorders

ABBREVIATIONS ACE = angiotensin-converting enzyme; $A G T$ = angiotensinogen; aSAH = aneurysmal subarachnoid hemorrhage; $A T 1$ = angiotensin II receptor Type $1 ; \mathrm{AT2}=$ angiotensin II receptor Type 2; CARAS = Cerebral Aneurysm Renin Angiotensin System; $\mathrm{CTA}=\mathrm{CT}$ angiography; $\mathrm{DCl}=$ delayed cerebral ischemia; DSA = digital subtraction angiography; eNOS = endothelial nitric oxide synthase; FDR = false discovery rate; HWE = Hardy-Weinberg equilibrium; $\mathrm{mRS}=$ modified Rankin Scale; PCR = polymerase chain reaction; RAS = renin-angiotensin system; SNP = single nucleotide polymorphism; VSMC = vascular smooth muscle cell.

SUBMITTED February 18, 2016. ACCEPTED April 11, 2016.

INCLUDE WHEN CITING Published online June 10, 2016; DOI: 10.3171/2016.4.JNS16409. 
$\mathrm{T}$ HE renin-angiotensin system (RAS) plays a crucial role in physiological vasorelaxation/vasoconstriction of the cerebral vasculature, vascular remodeling, and maintenance of arterial wall integrity. ${ }^{2,9,14}$ Compared with the normal arterial wall, cerebral aneurysms, in particular ruptured aneurysms, express significantly less angiotensin-converting enzyme (ACE) and angiotensin II receptor Type 1 (AT1), resulting in lack of vascular remodeling and thinning of the arterial wall under hemodynamic stress. ${ }^{23}$ Certain RAS genetic polymorphisms have been linked to aneurysmal subarachnoid hemorrhage (aSAH). In a meta-analysis, the II genotype of the ACE insertion/ deletion (I/D) polymorphism was associated with aSAH (OR 1.64, 95\% CI 1.24-2.17; $\mathrm{p}=0.009$ ) compared with controls. ${ }^{24}$ In the Cerebral Aneurysm Renin Angiotensin System (CARAS) study, allele $\mathrm{C}$ of the $\mathrm{X}$-linked angiotensin II receptor Type 2 (AT2) A/C single nucleotide polymorphism (SNP) demonstrated a dominant effect on aSAH (OR 3.48, 95\% CI 1.23-9.84; p = 0.0192) (Griessenauer et al., unpublished data, 2016). The significance of RAS genetic polymorphisms on the clinical course of aSAH, however, has not been studied. We sought to evaluate the association between common RAS polymorphisms of angiotensinogen (AGT), ACE, AT1, AT2, and clinical course after aSAH.

\section{Methods \\ Study Design}

A prospective trial evaluating the role of common RAS genetic polymorphisms in aSAH was performed at 2 academic institutions in the United States from September 2012 to January 2015. The study group included all patients who presented with aSAH. The diagnosis of SAH was established by admission CT scan or xanthochromia of cerebrospinal fluid (CSF). A ruptured cerebral aneurysm was confirmed by CT angiography (CTA) or digital subtraction angiography (DSA). Exclusion criteria were age younger than 19 years and any associated genetic syndrome that could explain the presence of a cerebral aneurysm (such as polycystic kidney disease, Ehler-Danlos syndrome Type 4, and Marfan syndrome) or systemic diseases (e.g., congestive heart failure and cirrhosis) that could interfere with RAS activity. All patients were enrolled within 72 hours of admission.

\section{General Management}

Patients presenting with aSAH were treated in accordance with contemporary standards of care in the United States: ICU monitoring, treatment of hydrocephalus, early (< 48 hours) intervention for aneurysm treatment, oral nimodipine, maintenance of euvolemia, and surveillance for clinical vasospasm and delayed cerebral ischemia (DCI). ${ }^{6}$ Following discharge from the ICU, patients are transferred to a ward with personnel trained in managing aSAH patients.

\section{Definition of Clinical Vasospasm and DCI}

Clinical vasospasm was defined as a new focal or global neurological deficit, or deterioration of at least 2 points on the Glasgow Coma Scale that is not explained by an- other clinical process including hydrocephalus, aneurysm rerupture, electrolyte disturbance, seizure, infection, fever, metabolic disturbance, cerebral edema, or surgical complication. Corroborating evidence of angiographic vasospasm was defined as arterial narrowing on CTA or DSA not due to atherosclerosis, catheter-induced vasospasm, or vessel hypoplasia. Additionally, vasospasm was diagnosed using transcranial Doppler ultrasound findings of a peak systolic middle cerebral artery of $>120 \mathrm{~cm} / \mathrm{sec}$ with a Lindegaard ratio of $>3$. CTA, DSA, and transcranial Doppler ultrasonography were performed at the discretion of the treating neurosurgeon. The diagnosis of clinical vasospasm was adjudicated by consensus of the study team and treated with hyperdynamic therapy as first line. ${ }^{7} \mathrm{Hy}-$ perdynamic therapy included avoidance of hypovolemia with a goal systolic blood pressure of greater than $160 \mathrm{~mm}$ $\mathrm{Hg}$, accomplished with either permissive hypertension or vasopressor therapy. Patients with clinical vasospasm refractory to medical treatment were treated in the endovascular suite at the discretion of the treating neurosurgeon.

CT scanning or MRI was routinely performed when the patient was transferred from the ICU to the ward. DCI was defined as low-density areas on CT that corresponded to a vascular distribution or an MR image demonstrating a hyperintense area on a diffusion-weighted imaging sequence with a corresponding hypointense apparent diffusion coefficient sequence correlate that corresponded with a vascular territory. Infarctions or contusions seen on postoperative Day 1 imaging were considered procedurally related and were not considered DCI.

\section{Outcome Measures in aSAH Patients}

Outcome measures included clinical vasospasm, DCI, mortality, and functional outcome at the time of discharge from the acute hospital setting, at 12 months, and at last follow-up using the modified Rankin Scale (mRS). Poor outcome was defined as death or severe disability (mRS Score 4-6). All outcome data were obtained blinded to the results of the genetic analysis. Functional outcome was assessed either in the clinic or via telephone interview with the patient or with a surrogate if the patient was unable to participate. ${ }^{3}$

\section{Laboratory and Genetic Evaluation}

A blood sample was obtained from all patients within 72 hours of admission for genetic evaluation and measurement of plasma ACE concentration. Common RAS genetic polymorphisms were detected using 5'exonuclease (TaqMan) genotyping assays (SNPs AGT C/T [rs699], AT1 A/C [rs5186], AT2 A/C [rs11091046], and G/A [rs1403543]) and restriction fragment length polymorphism analysis (ACE I/D [rs4340]). Commercial TaqMan assays were designed and performed according to the vendor (Thermo Fisher Scientific Inc.). Approximately $10 \%$ of the DNA samples were randomly selected to test reproducibility of TaqMan assays. All of the replication samples produced concordant genotypes. For the ACE I/D polymorphism, a polymerase chain reaction (PCR) based gel assay was used for the first round of genotyping with a second-round replication performed using an 


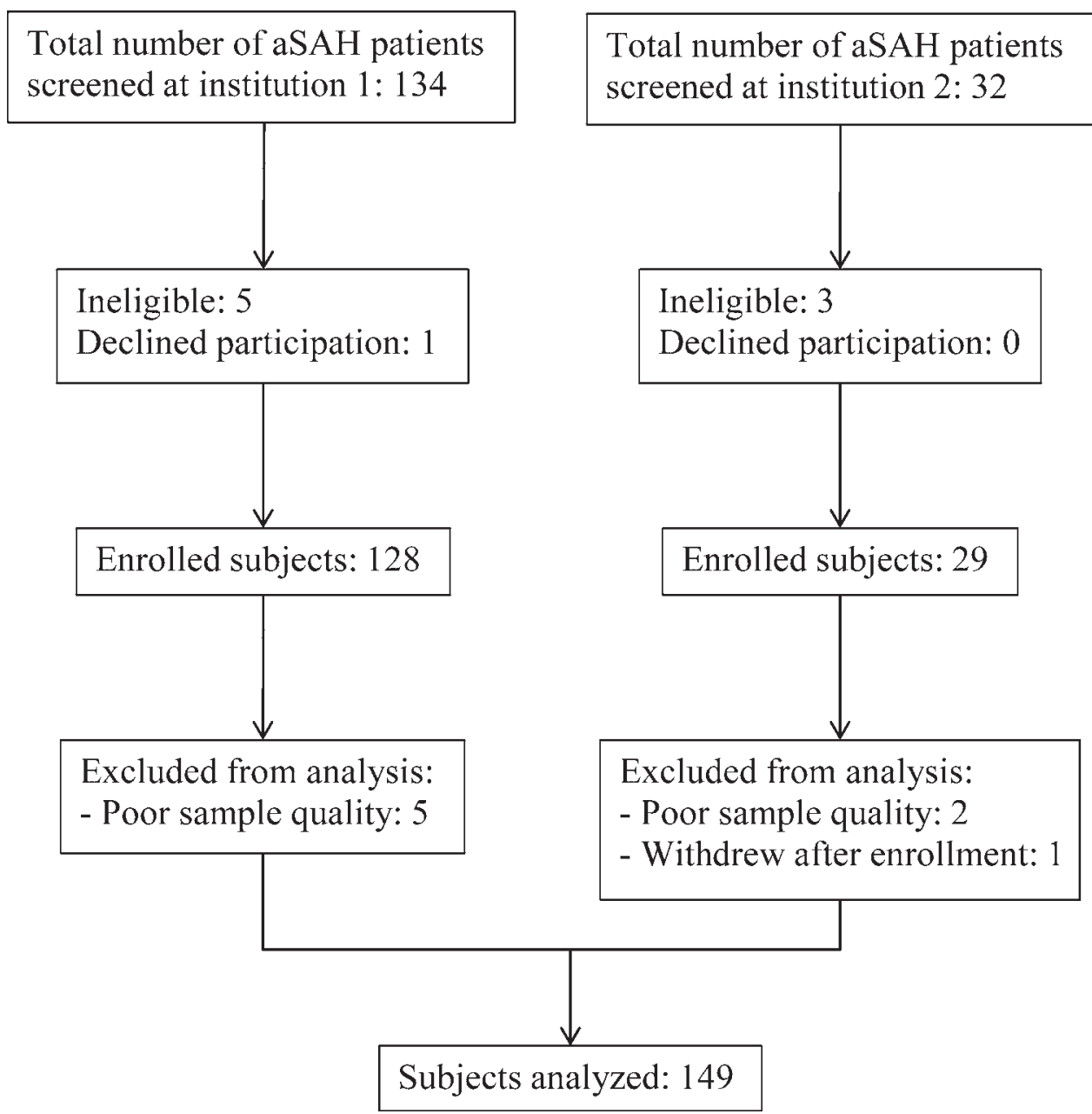

FIG. 1. Flowchart of participant enrollment.

amplification reaction containing $5 \mathrm{ng} / \mu \mathrm{l}$ of genomic DNA, $2 \mu \mathrm{M}$ of forward and reverse primers in a FailSafe Premix B 1× reaction (Epicentre). PCRs were performed in a C100 Touch Thermocycler (Bio-Rad), using cycling conditions as follows: 30 cycles with denaturation at $94^{\circ} \mathrm{C}$ for 1 minute, annealing at $58^{\circ} \mathrm{C}$ for 1 minute, and extension at $72^{\circ} \mathrm{C}$ for 2 minutes. PCR primers were as follows: forward primer, sense oligo 5'CTGGAGACCACTCCC ATCCTTCT3'; reverse primer, 5'GATGTGGCCATCA CATTCGTCAGAT3'. PCR products were resolved using the QIAxcel system (QIAGEN) to discriminate $490 \mathrm{bp}$ (insertion) from $202 \mathrm{bp}$ (deletion) alleles.

\section{Statistical Analysis}

Genotype frequencies for the individual SNPs and the ACE I/D were analyzed for all patients and then by age (patients $\leq 55$ years or $>55$ years; 55 years was the median age in the cohort). Sample size calculation was performed. Assuming a 50\% rate of clinical vasospasm, with an alpha of 0.05 and power of $90 \%$, a total sample size of 146 is needed to detect genotype associated with a $25 \%$ rate of vasospasm. Hardy-Weinberg equilibrium (HWE) was assessed using the chi-square test in aSAH patients and controls separately. To identify potential confounding variables, we tested for associations between patient characteristics, outcome measures, and genotype. Categorical variables and numerical variables were compared using Fisher's exact test and 1-way ANOVA, respectively. The association of genotype and outcome measures was tested using binary logistic regression with a standard method for dominant and recessive models. Multivariate logistic regression was subsequently performed to control for race and hypertension for all comparisons with $\mathrm{p}<0.1$. Adjustment of the false discovery rate (FDR) associated with multiple comparisons was performed using the Benjamini-Hochberg procedure. ${ }^{1}$ The FDR was set to $10 \%$. Unless specifically indicated, associations were not statistically significant after FDR correction.

\section{Results}

Between September 2012 and February 2015, 166 aSAH patients were screened for inclusion. Nine patients who were screened were excluded. A total of 157 aSAH patients were enrolled. One aSAH patient withdrew from the study, and blood samples of 7 aSAH patients could not be processed (Fig. 1). 
TABLE 1. Patient characteristics and outcome measures in 149 patients with aSAH

\begin{tabular}{|c|c|}
\hline Variable & Value $^{*}$ \\
\hline \multicolumn{2}{|l|}{ Patient characteristics } \\
\hline Mean age in yrs & $54.9 \pm 12.5$ \\
\hline \multicolumn{2}{|l|}{ Race } \\
\hline White & $85(57.0)$ \\
\hline African American & $60(40.3)$ \\
\hline Other & $4(2.7)$ \\
\hline \multicolumn{2}{|l|}{ Sex } \\
\hline Male & $35(23.5)$ \\
\hline Female & $114(76.5)$ \\
\hline Ischemic vascular disease & $13(8.7)$ \\
\hline Hypertension & $90(60.4)$ \\
\hline ACE inhibitor or ARB & $42(28.2)$ \\
\hline \multicolumn{2}{|l|}{ Smoker } \\
\hline Never & $58(38.9)$ \\
\hline Former & $18(12.1)$ \\
\hline Current & $73(49.0)$ \\
\hline Unruptured aneurysm(s) & $32(21.5)$ \\
\hline Family history of intracranial aneurysms & $14(9.4)$ \\
\hline \multicolumn{2}{|l|}{ Aneurysm size in $\mathrm{mm}$} \\
\hline$<7$ & $93(62.4)$ \\
\hline $7-12$ & $49(32.9)$ \\
\hline $13-24$ & $6(4.0)$ \\
\hline$\geq 25$ & $1(0.7)$ \\
\hline \multicolumn{2}{|l|}{ Location } \\
\hline Anterior & $120(80.5)$ \\
\hline Posterior & $29(19.5)$ \\
\hline \multicolumn{2}{|l|}{ Hunt \& Hess grade } \\
\hline$|-|||$ & $115(77.2)$ \\
\hline IV \& V & $34(22.8)$ \\
\hline \multicolumn{2}{|l|}{ Fisher grade } \\
\hline $1-2$ & $23(15.4)$ \\
\hline $3-4$ & $126(84.6)$ \\
\hline \multicolumn{2}{|l|}{ Treatment† } \\
\hline Microsurgical clipping & $76(52.4)$ \\
\hline Endovascular treatment & $69(47.6)$ \\
\hline Rerupture & $7(4.7)$ \\
\hline \multicolumn{2}{|l|}{ Outcome measures } \\
\hline Clinical vasospasm & $34(22.8)$ \\
\hline $\mathrm{DCl}$ & $31(21.2)$ \\
\hline Mean ICU stay in days & $11.9 \pm 7.9$ \\
\hline Mean hospital stay in days & $15.9 \pm 11.3$ \\
\hline \multicolumn{2}{|l|}{ Discharge mRS score } \\
\hline $0-3$ & $92(61.7)$ \\
\hline $4-6$ & $57(38.3)$ \\
\hline \multicolumn{2}{|l|}{ mRS score at last follow-up } \\
\hline $0-3$ & $120(80.5)$ \\
\hline $4-6$ & $29(19.5)$ \\
\hline Mean length of follow-up in days & $249.7 \pm 187.8$ \\
\hline
\end{tabular}

ARB = angiotensin receptor blocker.

* Values are presented as the number of patients (\%) unless noted otherwise.

Mean values are presented as the mean \pm SD.

$\dagger$ Data were not available for 4 aneurysms.

$\ddagger$ Data were not available for 3 aneurysms.

\section{Patient Characteristics and Outcome Measures}

The mean age of aSAH patients was $54.9 \pm 12.5$ years (male/female ratio 1:3.26). The majority (62.4\%) of ruptured aneurysms were smaller than $7 \mathrm{~mm}$ in size and were located in the anterior circulation (80.5\%). Favorable Hunt and Hess Grades I-III and Fisher CT Grades 1 and 2 were present in $77.2 \%$ and $15.4 \%$ of patients, respectively. Microsurgical clipping and endovascular treatment were performed in $52.4 \%$ and $47.6 \%$, respectively, and $4.7 \%$ of patients experienced rerupture of the aneurysm prior to treatment. Clinical vasospasm, DCI, and poor functional outcome at last follow-up occurred in $22.8 \%, 21.2 \%$, and $19.5 \%$ of patients, respectively (Table 1).

\section{Association of RAS Polymorphisms and Clinical Course}

Genotype frequencies of the individual SNPs and ACE I/D polymorphism were found to be in HWE. The Xlinked AT2 SNPs were in HWE in males and females.

\section{Angiotensinogen C/T Polymorphism (rs699)}

Univariable analysis showed statistically significant associations of genotype with race $(\mathrm{p}<0.0001)$ that persisted after FDR correction. The association with aneurysm size $(p=0.0226)$ was not significant after FDR correction (Table 2). In patients older than 55 years of age, genotype was associated with clinical vasospasm $(p=0.0369)$ and DCI ( $p=0.0173)$; however, this was not significant after FDR correction (Table 3).

In logistic regression, there was a statistically significant recessive effect of the C allele (CC vs CT + TT) on aneurysm size $(<7 \mathrm{~mm}$ vs $\geq 7 \mathrm{~mm})(\mathrm{OR} 2.63,95 \% \mathrm{CI}$ $1.06-6.51 ; p=0.0367)$ after controlling for race and performing FDR correction (Table 4). There was also a nonsignificant dominant effect of the T allele (CC vs CT + TT) on clinical vasospasm (OR 4.62, 95\% CI 0.719-29.7; $\mathrm{p}=0.107)$ and DCI (OR 9.54, 95\% CI 0.878-105; $\mathrm{p}=$ 0.0651) in patients older than 55 years after controlling for race and hypertension (Table 4).

\section{Angiotensin Converting Enzyme (ACE) I/D Polymorphism (rs4340)}

Univariable analysis showed a trend toward a statistically significant association of genotype with Hunt and Hess grade $(p=0.0682)$ (Table 2$)$ and mRS score at last follow-up ( $\mathrm{p}=0.129$ ) (Table 3). The ACE concentration was the lowest in aSAH patients with II genotype (17.6 \pm $8.0 \mathrm{U} / \mathrm{L})(\mathrm{p}=0.0195)($ Table 2).

In logistic regression, there was a recessive effect of the I allele (II vs ID + DD) on Hunt and Hess grade (OR $2.76,95 \%$ CI 1.17-6.50; $\mathrm{p}=0.0206$ ) that remained significant after FDR correction. After controlling for Hunt and Hess grade, the effect on mRS was no longer present (OR $1.54,95 \%$ CI $0.498-4.74, \mathrm{p}=0.455$ ). In patients 55 years or younger, there was a similar effect of the I allele on clinical vasospasm and DCI (OR 3.63, 95\% CI 1.04-12.7; $\mathrm{p}=0.0439)$; this remained significant after FDR correction (Table 4).

Angiotensin 2 Type 1 Receptor (AT1) A/C Polymorphism (rs5186)

No genotype associations with patient characteris- 


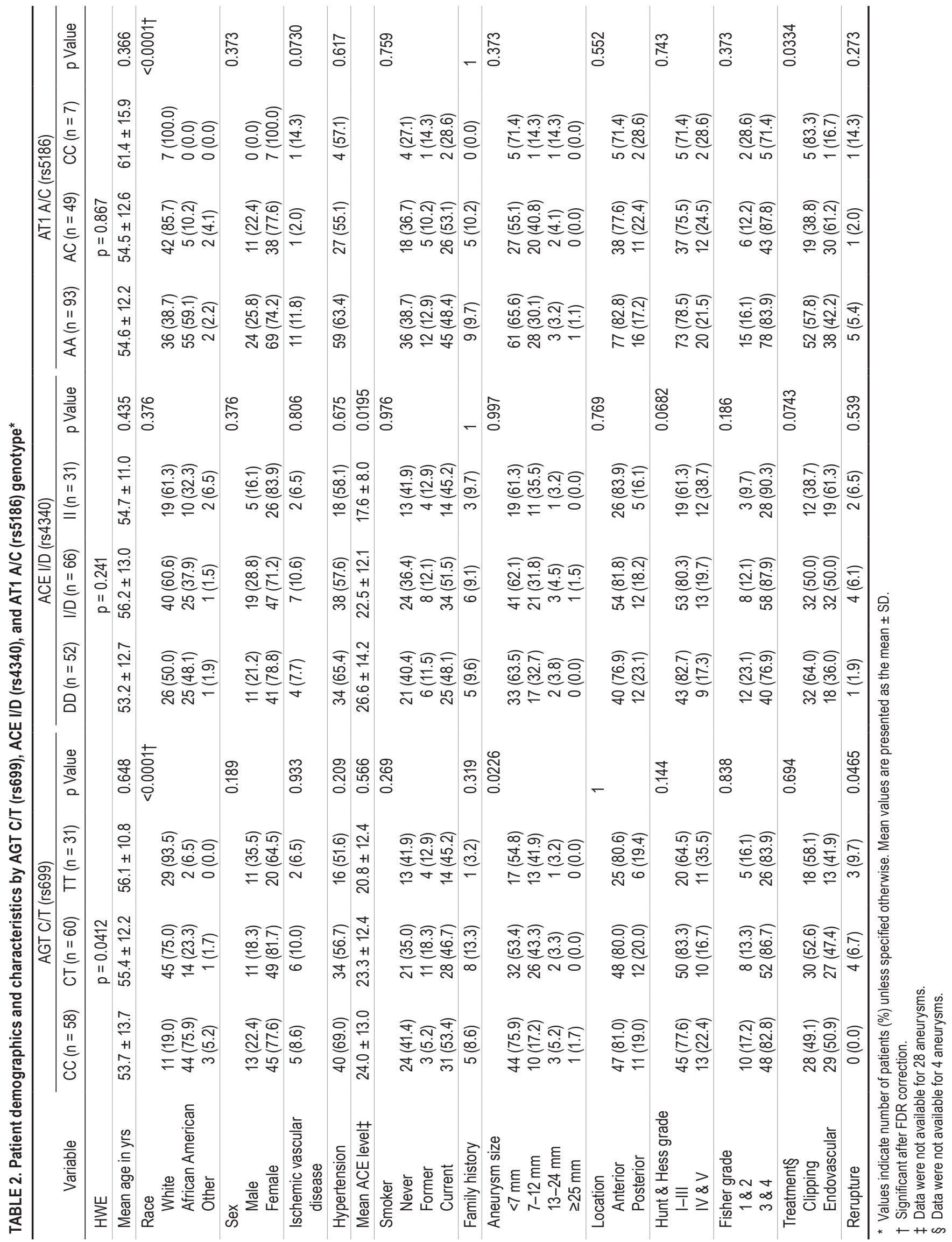




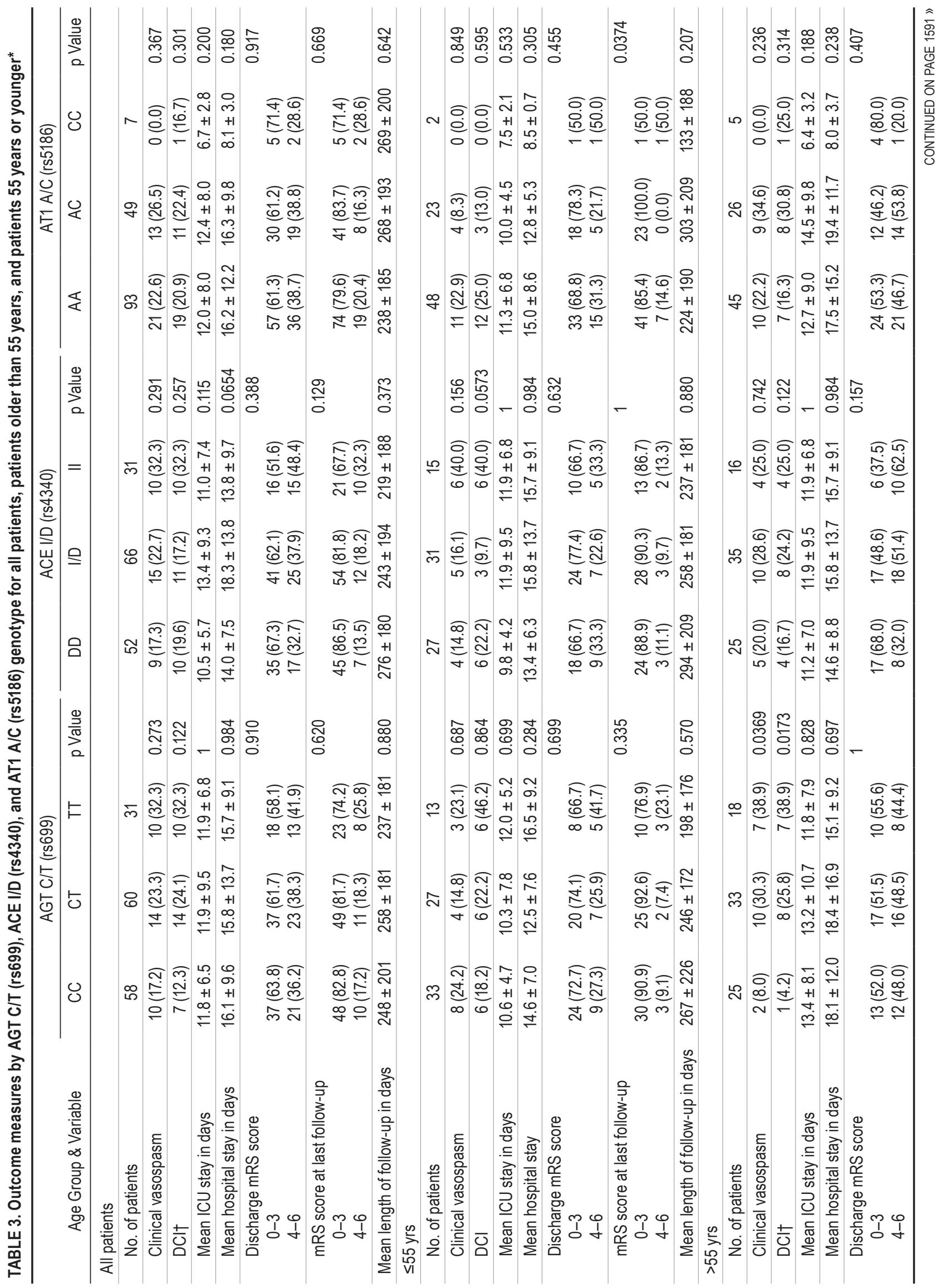




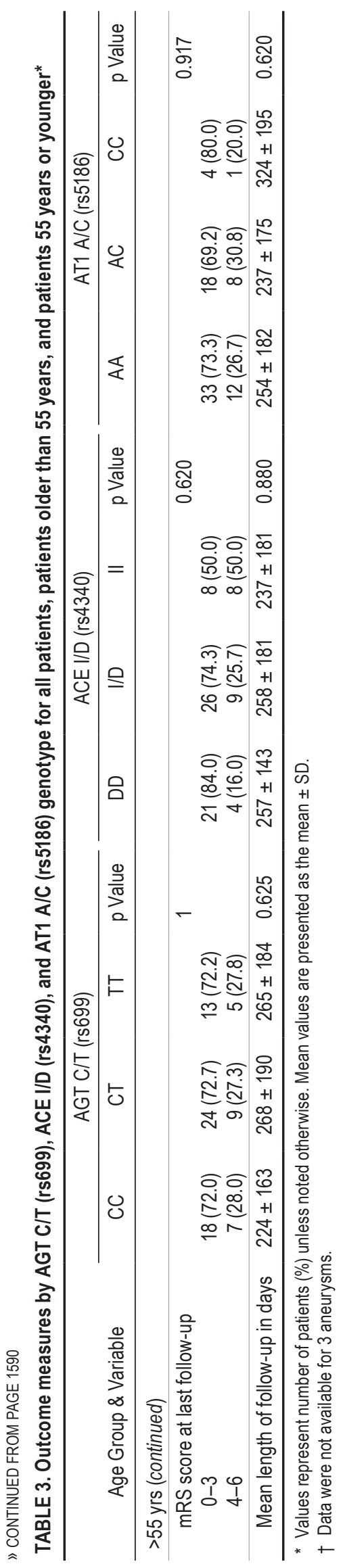

tics and outcome measures were identified in univariable analysis for the entire patient group and those older than 55 years. In patients 55 years or younger, genotype was associated with $\mathrm{mRS}$ score at last follow-up $(\mathrm{p}=0.0374)$ (Table 3). This association was not statistically significant following FDR correction or logistic regression.

\section{AT2 A/C Polymorphism (rs11091046)}

No genotype associations with aSAH were identified in univariable analysis for the entire patient group and patients 55 years or younger (Table 5). In patients older than 55 years, genotype was associated with clinical vasospasm $(p=0.0277)$ and DCI $(p=0.0332)$, but this was not significant after FDR correction (Table 6).

In logistic regression, there was a recessive effect of allele $\mathrm{A}\left(\mathrm{X}^{\mathrm{A}} \mathrm{X}^{\mathrm{A}}+\mathrm{X}^{\mathrm{A}} \mathrm{Y}\right.$ vs $\left.\mathrm{X}^{\mathrm{A}} \mathrm{X}^{\mathrm{C}}+\mathrm{X}^{\mathrm{C}} \mathrm{X}^{\mathrm{C}}+\mathrm{X}^{\mathrm{C}} \mathrm{Y}\right)$ on clinical vasospasm (OR 3.04, 95\% CI 0.990-9.30; $\mathrm{p}=0.0523$ ) and DCI (OR 4.70, 95\% CI 1.43-15.4; $\mathrm{p}=0.0111)$; these findings remained significant after FDR correction (Table 4).

\section{AT2 G/A Polymorphism (rs1403543)}

No genotype associations with aSAH were identified in univariable analysis for the entire patient group and subgroups of patients $\leq 55$ years or younger and those older than 55 years (Tables 5 and 6).

\section{Discussion}

The CARAS ${ }^{10,11}$ study prospectively enrolled aSAH patients and controls to evaluate associations among common RAS genetic polymorphisms and identified a dominant effect of allele C of AT2 A/C SNP on aSAH in patients older than 55 years (Griessenauer et al., unpublished data, 2016). Whereas systemic RAS is a potent vasoregulator, local RAS plays a significant role in vascular remodeling. Selective association of this receptor polymorphism with aSAH suggests that antitrophic effects mediated through AT2 receptors on vascular smooth muscle cells (VSMCs) may be relevant to the formation and rupture of cerebral aneurysms. In the present study, there were significant associations of RAS polymorphisms with the clinical course of aSAH. The I allele of the ACE I/D polymorphism was associated with poor Hunt and Hess grade and subsequent poor functional outcome. Both the I allele of the ACE I/D polymorphism and the A allele of the AT2 A/C SNP demonstrated age-dependent associations with clinical vasospasm and DCI.

\section{Renin-Angiotensin Polymorphisms and Risk Factors for aSAH}

This study identified important associations for the AGT C/T and ACE I/D polymorphism and risk factors for aSAH. Patients homozygous for the $\mathrm{C}$ allele of the AGT $\mathrm{C} / \mathrm{T}$ polymorphism experienced a higher rate of rupture from aneurysms smaller than $7 \mathrm{~mm}$. Identification of risk factors for rupture of small aneurysms is critically important, as size remains a controversial issue in aneurysm management. While the average size of ruptured aneurysms measures 6 to $7 \mathrm{~mm},{ }^{15,21}$ natural history studies of unruptured aneurysms indicate a benign course for small aneurysms. The ISUIA (International Study of Unrup- 
TABLE 4. Dominant and recessive effects of RAS polymorphisms on neurological status at presentation and outcome measures in aSAH

\begin{tabular}{|c|c|c|c|c|c|}
\hline Polymorphism & Outcome & Age & Effect & OR $(95 \% \mathrm{Cl})$ & p Value* \\
\hline AGT G/A (rs699) & Aneurysm size $<7 \mathrm{~mm}$ & All patients & $\begin{array}{l}\text { Recessive effect of the } \mathrm{C} \text { allele } \\
\quad(\mathrm{CC} \text { vs CT + TT) }\end{array}$ & $2.69(1.86-3.91) \dagger$ & 0.0077 \\
\hline AGT G/A (rs699) & Clinical vasospasm & $>55 \mathrm{yrs}$ & $\begin{array}{l}\text { Dominant effect of the T allele } \\
\text { (TT + CT vs CC) }\end{array}$ & $5.75(1.21-27.4) \ddagger$ & 0.0278 \\
\hline AGT G/A (rs699) & $\mathrm{DCl}$ & $>55 \mathrm{yrs}$ & $\begin{array}{l}\text { Dominant effect of the T allele } \\
\qquad(\mathrm{TT}+\mathrm{CT} \text { vs CC) }\end{array}$ & $10.1(1.25-82.0) \S$ & 0.0300 \\
\hline ACE I/D (rs4340) & Hunt \& Hess grade & All patients & $\begin{array}{l}\text { Recessive effect of the I allele } \\
\quad(I I \text { vs ID + DD) }\end{array}$ & $2.76(1.17-6.50)$ & 0.0206 \\
\hline ACE I/D (rs4340) & mRS score at last follow-up & All patients & $\begin{array}{l}\text { Recessive effect of the I allele } \\
\quad(I I \text { vs ID + DD) }\end{array}$ & $2.48(1.01-6.10) \rrbracket$ & 0.0475 \\
\hline ACE I/D (rs4340) & Hunt \& Hess grade & $>55 \mathrm{yrs}$ & $\begin{array}{l}\text { Recessive effect of the I allele } \\
\quad \text { (II vs ID + DD) }\end{array}$ & $4.00(1.25-12.8)$ & 0.0198 \\
\hline ACE I/D (rs4340) & mRS score at last follow-up & $>55 \mathrm{yrs}$ & $\begin{array}{l}\text { Recessive effect of the I allele } \\
\quad \text { (II vs ID + DD) }\end{array}$ & $3.61(1.14-11.5)^{\star *}$ & 0.0294 \\
\hline ACE I/D (rs4340) & Clinical vasospasm & $\leq 55 \mathrm{yrs}$ & $\begin{array}{l}\text { Recessive effect of the I allele } \\
\text { (II vs ID + DD) }\end{array}$ & $3.63(1.04-12.7)$ & 0.0439 \\
\hline ACE I/D (rs4340) & $\mathrm{DCl}$ & $\leq 55 \mathrm{yrs}$ & $\begin{array}{l}\text { Recessive effect of the I allele } \\
\text { (II vs ID + DD) }\end{array}$ & $3.63(1.04-12.7)$ & 0.0439 \\
\hline AT2 A/C (rs11091046) & Clinical vasospasm & $>55 \mathrm{yrs}$ & $\begin{array}{l}\text { Recessive effect of the A allele } \\
\qquad\left(X^{A} X^{A}+X^{A} Y \text { vs } X^{A} X^{C}+X^{C} X^{C}+X^{C} Y\right)\end{array}$ & $3.04(0.990-9.30)$ & 0.0523 \\
\hline AT2 A/C (rs11091046) & $\mathrm{DCl}$ & $>55 \mathrm{yrs}$ & $\begin{array}{l}\text { Recessive effect of the } A \text { allele } \\
\qquad\left(X^{A} X^{A}+X^{A} Y \text { vs } X^{A} X^{C}+X^{C} X^{C}+X^{C} Y\right)\end{array}$ & $4.70(1.43-15.4)$ & 0.0111 \\
\hline
\end{tabular}

* $p$ values significant after FDR correction.

† After adjusting for race, OR $2.63,95 \% \mathrm{Cl} 1.06-6.51 ; p=0.0367$, statistically significant.

$\ddagger$ After adjusting for race and hypertension, OR $4.62,95 \% \mathrm{Cl} 0.719-29.7 ; p=0.107$.

$\S$ After adjusting for race and hypertension, OR $9.54,95 \% \mathrm{Cl} 0.878-105 ; p=0.0651$.

II After adjusting for Hunt and Hess grade, OR 1.54, 95\% Cl 0.498-4.74; $p=0.455$.

** After adjusting for Hunt and Hess grade, OR 2.12, 95\% Cl 0.519-8.71; $p=0.295$.

tured Intracranial Aneurysms) reported a 5-year cumulative rupture rate of $1.5 \%$ and $0 \%$ for aneurysms smaller than $7 \mathrm{~mm}$ in the anterior circulation in patients with and without a history of SAH, respectively. ${ }^{31}$ The UCAS (Natural Course of Unruptured Cerebral Aneurysm Study of Japan) found annual rupture rates of $0.36 \%$ and $0.5 \%$ for aneurysms measuring 3-4 $\mathrm{mm}$ and 5-6 $\mathrm{mm}$, respectively. ${ }^{22}$ Both studies, however, were likely influenced by treatment selection bias. In UCAS, $69.7 \%$ of all aneurysms selected for elective treatment were smaller than $7 \mathrm{~mm}$ in maximum diameter. Factors currently considered in the decision to electively treat small aneurysms include female sex, young age, smoking, hypertension, history of aSAH, and aneurysm morphology.5,19 Whereas a family history of aSAH is a recognized risk factor, specific genetic information is not currently part of routine aneurysm management. Associations between genetic risk factors and aneurysm size at the time of rupture have been investigated. Of the 7 SNPs found to be associated with aneurysms in genome-wide association studies, none exerted influence on the aneurysm size at rupture. ${ }^{18}$ These genetic risk loci, however, only explain approximately $5 \%$ of the genetic risk for aneurysms $\mathrm{s}^{18}$ and did not include the AGT $\mathrm{C} / \mathrm{T}$ polymorphism. The AGT TT genotype is associated with hypertension in women and increased plasma AGT levels. ${ }^{25,27}$ The CC genotype may result in decreased AGT levels and subsequently decreased local RAS activity. Re- duced vascular remodeling due to locally downregulated RAS may lead to thinning and weakening of the arterial wall and aneurysm rupture at a smaller size. Another SNP linked to aneurysm size at rupture is the endothelial nitric oxide synthase (eNOS) T786C SNP. Patients homozygous for either $\mathrm{T}$ or $\mathrm{C}$ alleles experienced rupture of smaller aneurysms compared with heterozygotes. ${ }^{17}$ Interestingly, neither AGT C/T (Griessenauer et al., unpublished data, 2016) nor eNOS was associated with aSAH as compared with controls. ${ }^{17}$

The present study also found recessive effects of the I allele of the ACE I/D polymorphism on Hunt and Hess grade and subsequent functional outcome. Whereas the II genotype was found to be associated with aSAH, ${ }^{16,24,27}$ no such effect was found in CARAS. ${ }^{24}$ The II genotype is associated with the lowest ACE activity; thus, bradykinin levels are predicted to be increased, resulting in more vascular dilation and less VSMC proliferation locally. ${ }^{32}$ While aneurysm formation and rupture may be associated with decreased local RAS activity, decreased systemic RAS activity linked to the I allele may be responsible for the association with poor Hunt and Hess grade. In the acute setting of the aneurysm rupture there is a sudden surge in intracranial pressure and deterioration of cerebral autoregulation. ${ }^{4}$ Thus, systemic vasoregulation is critical for maintaining cerebral perfusion. Decreased systemic RAS activity associated with the I allele may complicate main- 







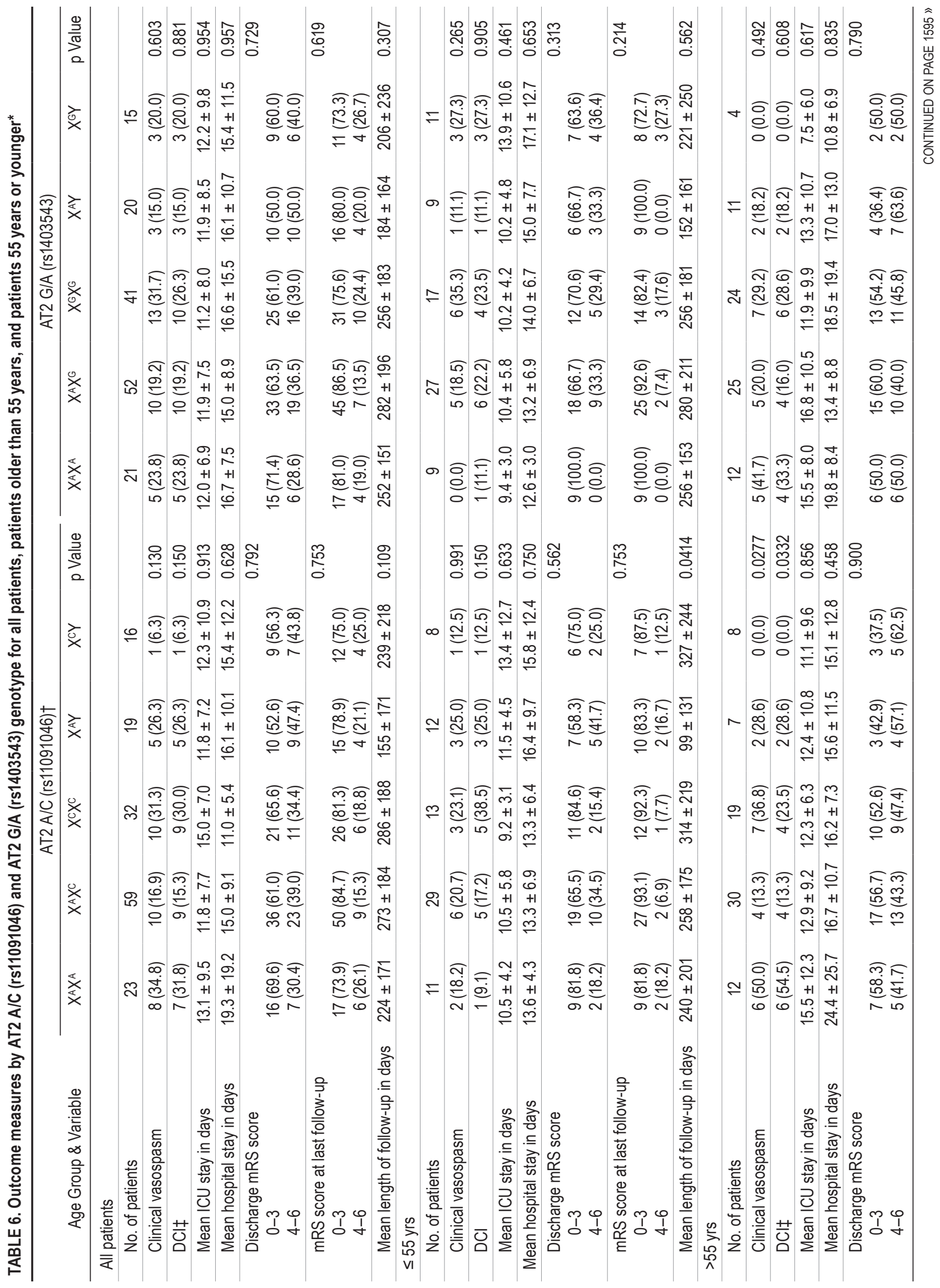




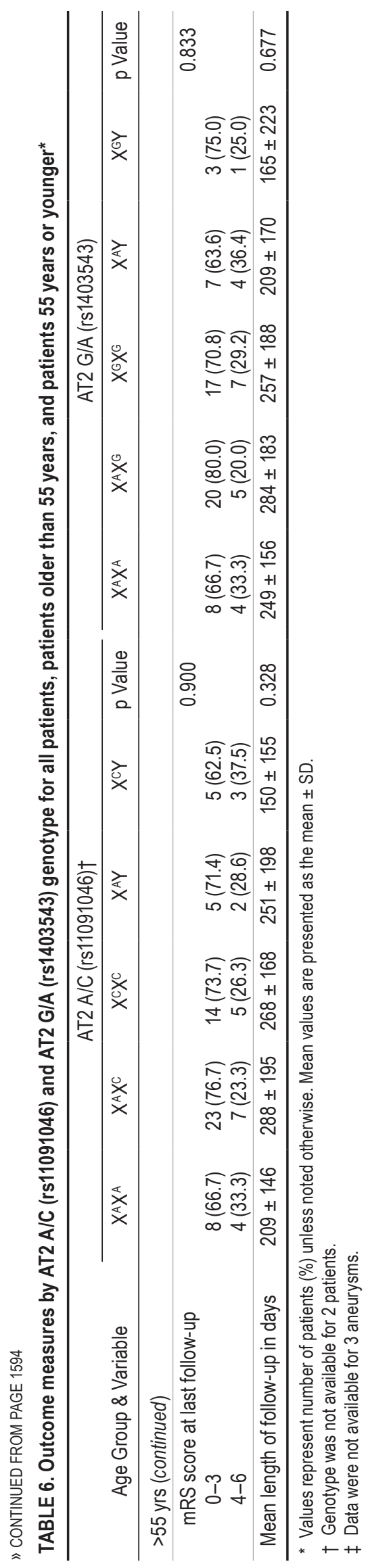

tenance of cerebral perfusion and render the patient more susceptible to transient ischemia. This process may be exacerbated if the patient also suffers from hydrocephalus. The ACE I/D genotype was not independently associated with functional outcome, and poor functional outcome associated with the II genotype may be an effect of poor Hunt and Hess grade.

\section{Clinical Vasospasm and DCI}

Once the aneurysm is secured, DCI represents the most significant threat to a patient's clinical course. The current study identified important associations among RAS polymorphisms, clinical vasospasm, and DCI. While there were recessive effects of the I allele of the ACE I/D polymorphism in patients 55 years and younger, DCI in older patients was associated with the A allele of the AT2 A/C SNP. Both systemic and local RAS modulation may be involved in the development of DCI. Younger patients homozygous for the I allele may have difficulties mounting a systemic response to cerebral vasospasm, making them susceptible to DCI. The C allele of the AT2 SNP has been associated with trophic effects in the local RAS. ${ }^{8}$ While antitrophic effects on the VSMCs appear to predispose to aneurysm formation and rupture, overactivation of the VSMCs via the local AT2 receptor may exacerbate vasospasm and DCI. Activation of RAS in aSAH occurs around 4 to 6 days after aneurysm rupture as a response to sodium and water loss triggered by brain and atrial natriuretic peptides. ${ }^{20}$ This delayed RAS activation coincides with the onset of cerebral vasospasm, and it is plausible that in an interrelated system of enzymes and substrates such as RAS, a change in activity or level of individual components may alter the susceptibility for clinical vasospasm and DCI. Other genetic polymorphisms associated with vasospasm and DCI include endothelin- $1,{ }^{12}$ cystathionine b-synthase, ${ }^{13}$ eNOS, ${ }^{26,29}$ haptoglobin, ${ }^{26}$ and plasminogen activator inhibitor- $1 .{ }^{30}$ How these polymorphisms relate to the RAS-related effects identified in the present study remains to be determined.

\section{Limitations and Future Directions}

There are limitations to the present study. Patients who died prior to or soon after admission could not be consented to participate in the study. Thus, findings only apply to aSAH patients who survived the acute phase. Methodologically, multiple testing may find false-positive associations of genotypes with outcome measures by chance. In an effort to reduce the rate of false-positive findings, we performed adjustment of the FDR associated with multiple comparisons. ${ }^{1}$ The population and sample size was relatively small, a recognized limitation of studying rare disease processes. The patient population represented individuals presenting to a tertiary referral center in the eastern and southeastern United States, thus limiting broad generalizability. Lastly, the associations identified in the present study occurred on the genomic level. Future work will focus on gene expression profiles and protein activity associated with the polymorphism investigated to delineate the precise mechanism by which RAS polymorphisms may affect the clinical course after aSAH. 


\section{Conclusions}

The RAS appears to play a role in the clinical course of aSAH patients. Both the ACE I/D polymorphism and the AT2 A/C SNP were associated an age-dependent risk of DCI, whereas only the ACE I/D polymorphism was associated with poor clinical grade at presentation. Further studies are required to elucidate the relevant pathophysiology and its potential implication in treatment of patients with aSAH.

\section{Acknowledgments} dation

This study received the support of the Brain Aneurysm Foun-

\section{References}

1. Benjamini Y, Hochberg Y: Controlling the false discovery rate: a practical and powerful approach to multiple testing. J R Stat Soc Series B Stat Methodol 57:289-300, 1995

2. Brooks DP, Ruffolo RR: Functions mediated by peripheral angiotensin II receptors, in Ruffolo RR (ed): Angiotensin II Receptors, Vol 1: Molecular Biology, Biochemistry, Pharmacology and Clinical Perspectives. Boca Raton, FL: CRC Press, 1994, pp 71-102

3. Bruno A, Akinwuntan AE, Lin C, Close B, Davis K, Baute V, et al: Simplified modified Rankin scale questionnaire: reproducibility over the telephone and validation with quality of life. Stroke 42:2276-2279, 2011

4. Calviere L, Nasr N, Arnaud C, Czosnyka M, Viguier A, Tissot B, et al: Prediction of delayed cerebral ischemia after subarachnoid hemorrhage using cerebral blood flow velocities and cerebral autoregulation assessment. Neurocrit Care 23:253-258, 2015

5. Chalouhi N, Zanaty M, Whiting A, Yang S, Tjoumakaris S, Hasan D, et al: Safety and efficacy of the Pipeline Embolization Device in 100 small intracranial aneurysms. J Neurosurg 122:1498-1502, 2015

6. Connolly ES Jr, Rabinstein AA, Carhuapoma JR, Derdeyn CP, Dion J, Higashida RT, et al: Guidelines for the management of aneurysmal subarachnoid hemorrhage: a guideline for healthcare professionals from the American Heart Association/American Stroke Association. Stroke 43:1711-1737, 2012

7. Dankbaar JW, Slooter AJ, Rinkel GJ, Schaaf IC: Effect of different components of triple-H therapy on cerebral perfusion in patients with aneurysmal subarachnoid haemorrhage: a systematic review. Crit Care 14:R23, 2010

8. Deinum J, van Gool JM, Kofflard MJ, ten Cate FJ, Danser AH: Angiotensin II type 2 receptors and cardiac hypertrophy in women with hypertrophic cardiomyopathy. Hypertension 38:1278-1281, 2001

9. Drenjancevic-Peric I, Phillips SA, Falck JR, Lombard JH: Restoration of normal vascular relaxation mechanisms in cerebral arteries by chromosomal substitution in consomic SS.13BN rats. Am J Physiol Heart Circ Physiol 289:H188H195, 2005

10. Foreman PM, Chua M, Harrigan MR, Fisher WS III, Tubbs RS, Shoja MM, et al: Antifibrinolytic therapy in aneurysmal subarachnoid hemorrhage increases the risk for deep venous thrombosis: A case-control study. Clin Neurol Neurosurg 139:66-69, 2015

11. Foreman PM, Chua MH, Harrigan MR, Fisher WS III, Vyas NA, Lipsky RH, et al: Association of nosocomial infections with delayed cerebral ischemia in aneurysmal subarachnoid hemorrhage. J Neurosurg [epub ahead of print February 12, 2016. DOI: 10.3171/2015.10.JNS151959]

12. Gallek M, Alexander S, Crago E, Sherwood P, Horowitz M, Poloyac S, et al: Endothelin-1 and endothelin receptor gene variants and their association with negative outcomes following aneurysmal subarachnoid hemorrhage. Biol Res Nurs 15:390-397, 2013

13. Grobelny BT, Ducruet AF, DeRosa PA, Kotchetkov IS, Zacharia BE, Hickman ZL, et al: Gain-of-function polymorphisms of cystathionine $\beta$-synthase and delayed cerebral ischemia following aneurysmal subarachnoid hemorrhage. J Neurosurg 115:101-107, 2011

14. Haberl RL, Decker-Hermann PJ, Hermann K: Effect of renin on brain arterioles and cerebral blood flow in rabbits. J Cereb Blood Flow Metab 16:714-719, 1996

15. Harrigan M, Deveikis J: Intracranial aneurysms and subarachnoid hemorrhage, in Handbook of Cerebrovascular Disease and Neurointerventional Technique. New York: Humana Press, 2009, p 443

16. Keramatipour M, McConnell RS, Kirkpatrick P, Tebbs S, Furlong RA, Rubinsztein DC: The ACE I allele is associated with increased risk for ruptured intracranial aneurysms. J Med Genet 37:498-500, 2000

17. Khurana VG, Sohni YR, Mangrum WI, McClelland RL, O'Kane DJ, Meyer FB, et al: Endothelial nitric oxide synthase T-786C single nucleotide polymorphism: a putative genetic marker differentiating small versus large ruptured intracranial aneurysms. Stroke 34:2555-2559, 2003

18. Kleinloog R, van 't Hof FNG, Wolters FJ, Rasing I, van der Schaaf IC, Rinkel GJE, et al: The association between genetic risk factors and the size of intracranial aneurysms at time of rupture. Neurosurgery 73:705-708, 2013

19. Korja M, Lehto H, Juvela S: Lifelong rupture risk of intracranial aneurysms depends on risk factors: a prospective Finnish cohort study. Stroke 45:1958-1963, 2014

20. Lo BWY, Fukuda H, Nishimura Y, Macdonald RL, Farrokhyar F, Thabane L, et al: Pathophysiologic mechanisms of brain-body associations in ruptured brain aneurysms: A systematic review. Surg Neurol Int 6:136, 2015

21. McDougall CG, Spetzler RF, Zabramski JM, Partovi S, Hills NK, Nakaji P, et al: The Barrow Ruptured Aneurysm Trial. J Neurosurg 116:135-144, 2012

22. Morita A, Kirino T, Hashi K, Aoki N, Fukuhara S, Hashimoto $\mathrm{N}$, et al: The natural course of unruptured cerebral aneurysms in a Japanese cohort. N Engl J Med 366:2474-2482, 2012

23. Ohkuma H, Suzuki S, Fujita S, Nakamura W: Role of a decreased expression of the local renin-angiotensin system in the etiology of cerebral aneurysms. Circulation 108:785787, 2003

24. Peck G, Smeeth L, Whittaker J, Casas JP, Hingorani A, Sharma P: The genetics of primary haemorrhagic stroke, subarachnoid haemorrhage and ruptured intracranial aneurysms in adults. PLoS One 3:e3691, 2008

25. Petkeviciene J, Klumbiene J, Simonyte S, Ceponiene I, Jureniene K, Kriaucioniene V, et al: Physical, behavioural and genetic predictors of adult hypertension: the findings of the Kaunas Cardiovascular Risk Cohort study. PLoS One 9:e109974, 2014

26. Rosalind Lai PM, Du R: Role of genetic polymorphisms in predicting delayed cerebral ischemia and radiographic vasospasm after aneurysmal subarachnoid hemorrhage: a metaanalysis. World Neurosurg 84:933-941, 941.e1-941.e2, 2015

27. Sethi AA, Nordestgaard BG, Agerholm-Larsen B, Frandsen E, Jensen G, Tybjaerg-Hansen A: Angiotensinogen polymorphisms and elevated blood pressure in the general population: the Copenhagen City Heart Study. Hypertension 37:875881,2001

28. Slowik A, Borratynska A, Pera J, Betlej M, Dziedzic T, Krzyszkowski T, et al: II genotype of the angiotensin-converting enzyme gene increases the risk for subarachnoid hemorrhage from ruptured aneurysm. Stroke 35:1594-1597, 2004

29. Starke RM, Kim GH, Komotar RJ, Hickman ZL, Black EM, Rosales MB, et al: Endothelial nitric oxide synthase gene 
single-nucleotide polymorphism predicts cerebral vasospasm after aneurysmal subarachnoid hemorrhage. J Cereb Blood Flow Metab 28:1204-1211, 2008

30. Vergouwen MDI, Frijns CJM, Roos YBWEM, Rinkel GJE, Baas F, Vermeulen M: Plasminogen activator inhibitor-1 4G allele in the $4 \mathrm{G} / 5 \mathrm{G}$ promoter polymorphism increases the occurrence of cerebral ischemia after aneurysmal subarachnoid hemorrhage. Stroke 35:1280-1283, 2004

31. Wiebers DO, Whisnant JP, Huston J III, Meissner I, Brown RD Jr, Piepgras DG, et al: Unruptured intracranial aneurysms: natural history, clinical outcome, and risks of surgical and endovascular treatment. Lancet 362:103-110, 2003

32. Yayama K, Okamoto H: Angiotensin II-induced vasodilation via type 2 receptor: role of bradykinin and nitric oxide. Int Immunopharmacol 8:312-318, 2008

\section{Disclosures}

The authors report no conflict of interest concerning the materi- als or methods used in this study or the findings specified in this paper.

\section{Author Contributions}

Conception and design: Griessenauer, Tubbs, Shoja. Acquisition of data: Griessenauer, Foreman, Vyas. Analysis and interpretation of data: Griessenauer, Foreman, Chua, Vyas, Lipsky, Lin, Iyer, Haridas, Chaudry, Malieva, Wilkins, Shoja. Drafting the article: Griessenauer, Foreman. Critically revising the article: Griessenauer, Tubbs, Foreman, Chua, Lipsky, Lin, Shoja. Reviewed submitted version of manuscript: Griessenauer, Tubbs, Foreman, Shoja. Approved the final version of the manuscript on behalf of all authors: Griessenauer. Statistical analysis: Griessenauer, Chua. Administrative/technical/material support: Griessenauer, Tubbs, Vyas, Lipsky, Lin, Iyer, Haridas, Walters, Harrigan, Fisher, Shoja. Study supervision: Griessenauer, Walters, Harrigan, Fisher, Shoja.

\section{Correspondence}

Christoph J. Griessenauer, Division of Neurosurgery, Harvard Medical School, 110 Francis St., Ste. 3B, Boston, MA 02215. email: christoph.griessenauer@gmail.com. 